\title{
An evaluation of the effectiveness, facilitators and barriers of HIV Teen Clubs in achieving successful transition from teen to adult care in Blantyre, Malawi using the RE-AIM Framework.
}

Jerome Galagade ( $\boldsymbol{\sim}$ jgalagade@gmail.com )

University of Malawi College of Medicine https://orcid.org/0000-0002-4216-6506

Victor Mwapasa

University of Malawi College of Medicine

Research article

Keywords: Adolescent, HIV, AIDS, Adhérence, transition in care, Teen Club, challenges,

Posted Date: October 27th, 2020

DOI: https://doi.org/10.21203/rs.3.rs-39459/v2

License: (ㄷ) (1) This work is licensed under a Creative Commons Attribution 4.0 International License. Read Full License 


\section{Abstract}

Background: High attrition is a major problem for Adolescent Living with HIV (ALHIV) transitioning from pediatric to adult care. Although the HIV Teen Club model has been shown to promote antiretroviral (ARV) adherence and viral suppression among ALHIV, its effect on promoting transitioning ALHIV from pediatric to adult care has not been formally evaluated. We sought to estimate the proportion of ALHIV enrolled in HIV Teen Clubs who successfully transitioned to adult care within six months of the expected transition time and assess health facilities' fidelity in implementing HIV Teen Clubs' adult care transition protocols and factors associated with successful transition to adult Care.

Methods: From September 2018 to June 2019, we conducted a retrospective descriptive cohort study involving ALHIV attending HIV Teen Clubs in 14 primary health facilities in Blantyre, Malawi who had reached the recommended transition age of 18/19 years between July - December 2017. After obtaining consent, we reviewed their records to assess the proportion who successfully transitioned and also factors associated with transitioning. Checklist was used to ascertain fidelity of implementation of HIV Teen Club transition protocols. In-depth interviews were conducted with service providers (SP) in health facilities providing HIV Teen Club to get their perspectives on reasons for successful or unsuccessful transition.

Results: Of the 131 eligible ALHIV enrolled in the study, only $6.9 \%(n=9)$ successfully transitioned to adult care within six months of the expected transition time. The overall fidelity in implementing adult care transition protocols in health facilities was medium. There was an association between implementation fidelity and ALHIV successful transition with 2 out of 3 health facilities with high implementation fidelity also successfully transitioning ALHIV into adult care. Successful ALHIV transitioning was associated with urban location of health facilities and ownership of the facilities by NGOs. SPs reported that lack of training in transitioning ALHIV, lack of clear guidelines, inadequate human resource and refusal of ALHIV to transition were some of the health system factors negatively influencing successful transition.

Conclusions: Training and mentorship of SP in implementing transitioning protocols and adequately resourcing HIV teen clubs may improve their operations and facilitate successful transitioning.

\section{Background}

In 2016, there were an estimated 2.1 million adolescents living with HIV (ALHIV) aged 10-19 years, worldwide [1]. HIV is the second leading cause of adolescent morbidity and mortality worldwide and the leading cause in Africa[2]. In Malawi, by 2016, HIV prevalence was estimated at 3\% among adolescents aged 15-24 years[3]. In 2016, there were an estimated 93,000 ALHIV in Malawi, making Malawi one of the top 10 countries in terms of numbers of ALHIV[4]. Around 12,500 new HIV infections occurred among the youth aged 15-24 from Malawi in 2016 alone[4].

The Malawi HIV and AIDS Strategic Plan, 2015 - 2020 (NSP) recognizes the importance of adolescent HIV and AIDS interventions by prioritizing access to youth friendly health services as a strategy for reducing new infections among adolescents. Despite rolling out the NSP, in 2017 knowledge of HIV status was lower among young people aged 15-24 (49.8\%) than adults $(72.7 \%)$. In addition, ART initiation is lower for young people who know their positive HIV status (82.5\%) than adults (89.6 \%). Furthermore, HIV viral suppression is lower in HIV+ youths on ART (78.8\%) than in adults (91.2\%) [5]. To address this challenge, the NSP advocates interventions that support adherence to ART and psychosocial support among the youth[6].

Both good adherence and retention in care are a prerequisite to successful management of adolescents living with HIV[7]. However, there is a potential risk of ART disruptions when ALHIV transition from pediatric to adult-oriented medical care[8]. During this process, the ALHIV need to change location of ART services from the pediatric to the adult care clinics but also assume responsibility of their own health and disease management, raising new challenges for sustaining retention in the care continuum[9]. Indeed, high loss-to-follow-up rates have been noted among adolescents transition from teen to adult care in Canada and United States of America, ranging from 24\% - 55 \% [9-12]. Poor retention in care and ART adherence may lead to poor health outcomes and risks the emergence of HIV resistance, which is expensive to treat [13]. Despite these potential 
threats, most countries, including Malawi have no specific national guidelines or protocols for transitioning adolescents to adult care so as to ensure continuity of ART adherence[14].

The World Health Organization recommends differentiated service delivery (DSD) models, as a way of improving retention in care among ART clients. Differentiated care is a client-centered approach to patient care which is employed focusing on the preferences and expectations of people living with HIV[15]. The HIV Teen club model is an example of DSD for ALHIV who are on ART. HIV Teen clubs mostly operate within primary health facility, offering various services to ALHIV aged $10-19$ years, including clinical care, counselling, health education and entertainment [16]. The services are provided by a multi-disciplinary team which include clinicians, nurse, nursing aids, lay community health worker and others. Services are mostly provided outside schools to eliminate school absenteeism[16]. Figure 1 shows an illustration of HIV Teen Club model and the typical client flow chart. Teens collect their medications from nurses and are assessed for adherence and provided with individualized peer counselling and support as necessary. Teens that require additional clinical assessment are referred for a clinician visit. As per national guideline, a total of 10 key staffs ( 6 health workers, 4 administrative) are required per Teen Club.

The Teen Club model includes a transition of care component which prepares the adolescents to transition from pediatric to the adult ART clinic at the very same health facility[16]. This involves providing psychosocial education on transition for adolescents that are eligible to transition to adult care. These adolescents are taught specific topics on transition which include; psychosocial development, sexual \& reproductive health, beneficial disclosure, positive living among others[16].

By 2018 there were over 158 HIV teen clubs in 26 of the 28 districts in Malawi, but there were no formal government guidelines governing their operations.[14]. A study conducted at Zomba Central Hospital in Malawi showed that the odds of attrition were 3.7-times lower in ALHIV exposed to Teen Clubs than those who were not exposed [17]. This was consistent with studies conducted in high income countries [10,12,18,19] and in South Africa [20]. However, the Malawian and South African studies did not explore the clinical outcomes of these adolescents and did not identify the specific elements of the teen clubs which were critical in ensuring successful transitioning. Although many health facilities operate Teen Clubs, no formal evaluation has been conducted to assess the fidelity of implementation of teen club HIV care protocols. In this study, we estimated the proportion of eligible ALHIV enrolled in Teen Clubs in Blantyre who successfully transitioned to adult care within six months of the expected transition time, assessed the fidelity in the implementation of HIV Teen Clubs' adult care transition protocols in health facilities' and determined social and demographic characteristics of adolescents associated with successful transition from HIV Teen Clubs to adult care.

\section{Methods}

\section{Study Design}

The study used a mixed-methods approach, comprising of a quantitative retrospective descriptive cohort study and qualitative grounded theory study. The cohort study involved a retrospective review of records of a cohort of ALHIV attending HIV Teen Clubs. The records included clinic registers, individual patient master cards and Electronic Medical Records (EMR). In addition, an observation checklist was used to ascertain fidelity of implementation of HIV Teen Club transition protocols in 14 of the 21 primary health facilities that had HIV Teen Clubs in 2018, namely; Chikowa, Chileka, South Lunzu, Mpemba, Mlambe, Mdeka, Lirangwe, Chilomoni, Ndirande, Limbe, Bangwe, Madziabango, DREAM Sant'Egidio and Zingwangwa. The qualitative grounded theory [21]study involved In-depth Interviews (IDI) with health service providers (SPs)to get their perspectives on operating HIV Teen Clubs and reasons for successful or unsuccessful transition..

\section{Participants}

The following were the inclusion criteria: a) ALHIV who had reached 18/19 years old in July - Dec 2017 b) had completed secondary school c) Had stayed in the club for one and half years (having gone through the curriculum 3 times). The study also included SPs involved in Teen Clubs and ART treatment for more than one and half years. The study excluded health facilities that did not have HIV Teen Clubs, SP not in ART treatment service provision and facilities outside Blantyre. 


\section{Evaluation Methodology: The RE-AIM Framework}

The study used RE-AIM Framework to evaluate the effectiveness of the HIV Teen Club model [22]. This is an evaluation framework that systematically evaluates public health interventions using five domains, namely Reach, Effectiveness, Adoption, Implementation, and Maintenance and these are defined in Figure 2. A central tenet of the framework is that the ultimate impact of an intervention depended on the combined effects of the 5 evaluative domains. The framework provides scientists some degree of flexibility to select domains relevant to their study. [23].

Our study focused on the following 3 domains of the RE-AIM model informed by the research question:

1. Effectiveness was measured by estimating the proportion of eligible adolescents in Teen Clubs who have successfully transitioned to adult care in selected HIV Teen Clubs.

2. Implementation (Fidelity) was measured by assessing implementation of HIV Teen Clubs protocols that promote successful transition to adult care

3. Maintenance was measured by assessing factors (social, demographic, health system) associated with successful transition from HIV Teen Clubs to adult care.

\section{Sample size and Sampling technique}

We planned to select ALHIV from HIV Teen Club using probability proportion to size (PPS) sampling procedure. A sample size of 145 was calculated based on estimated ALHIV population in HIV Teen Club. The Cochran method [21] was used to calculate the sample size for estimation of proportion of adolescents successfully transitioning to adult care. The parameters used in this sample size estimation included: We estimated successful transition to adult care of 45\% [24] the standard normal (Z) value corresponding to $95 \%$ confidence interval and a precision of $+/-5 \%$. However, we were unable to reach our sample size due to poor record keeping and thus, the study enrolled 131 ALHIV. We also interviewed two purposefully-selected SPs per facility (i.e. Teen club Coordinator and a Nurse or Clinician), summing up to 28 SPs.

\section{Data Collection}

Literature review was conducted between September to December 2018 with data collection from January to June 2019. ALHIV data on ART number, sex, age, location, occupation, clinic visits, and date of joining Teen Club among adolescents eligible for transition between July-December 2017 was drawn from the HIV Teen Club register and further verified through extraction of related data in the health facility Electronic Medical Records (EMR) using a data extraction tool. We then extracted six months' data on prescribed clinical visit for ART refill in the EMR from January 2018 to June 2018 as a follow up period to ascertain those that remained in care after transition.

Structured observations were conducted in health facilities with active HIV Teen Clubs using an observation checklist adapted from HIV Teen Club Curriculum. To assess fidelity, the observation checklist focused on the main domains of intervention which include; clinical care, psychological support, Sexual Reproductive Health Services and Teen club operations. In-Depth interviews with SPs were conducted outside work hours and explored major themes of capacity building, effectiveness, transition challenges and sustainability. The In-Depth Interviews (IDI) were conducted in Chichewa or English, as preferred by the respondent and tape recorded.

\section{Data Analysis}

To calculate the proportion (95\% confidence interval) of eligible adolescents in Teen Clubs who successfully transitioned to adult care, descriptive analysis of retrospective quantitative data was performed. Successful transition was defined as adherence to prescribed scheduled visits on patient master card.

Table 1 shows the domain that were used to assess "fidelity" and the total scores for each domain. An item was scored as 1 when present or " 0 " when absent. The scores were summed up for each facility. Facilities were classified into the following 
three performance categories; low fidelity (score of less than 50 ), medium fidelity (a score of 50 but $<70$ ) and high fidelity (a score of 70 and above).

Table 1: HIV Teen Club Components that were evaluated

\begin{tabular}{|c|c|c|c|}
\hline $\begin{array}{l}\text { Components } \\
\text { evaluated }\end{array}$ & Description of elements & $\begin{array}{l}\text { Number of } \\
\text { elements } \\
\text { rated }\end{array}$ & $\begin{array}{l}\text { Total } \\
\text { expected } \\
\text { score per } \\
\text { domain }\end{array}$ \\
\hline $\begin{array}{l}\text { Domain one: } \\
\text { Teen Club } \\
\text { Operation }\end{array}$ & $\begin{array}{l}\text { 1. Designated place for ALHIV club meetings, } \\
\text { 2. Meeting place safe and private for ALHIV to meet and learn, } \\
\text { 3. Designated leaders available to facilitate meetings, } \\
\text { 4. Designated health care providers (Nurse, Clinician) available, } \\
\text { 5. Club has rules that govern its meetings, } \\
\text { 6. Club has attendance register, } \\
\text { 7. Club has a confidentiality agreement and a sign-in for } \\
\text { 8isitors, } \\
\text { 9. ALHIV are provided with transport reimbursement, }\end{array}$ & 9 & 9 \\
\hline $\begin{array}{l}\text { Domain two: } \\
\text { Capacity } \\
\text { Building }\end{array}$ & $\begin{array}{l}\text { 1. Club Facilitators were trained in teen club facilitation, } \\
\text { 2. Club Facilitators were trained in transition program, } \\
\text { 3. Minutes/records of the Teen Club that reflect that the } \\
\text { facilitators oriented the adolescents on the transition } \\
\text { process to adult care. }\end{array}$ & 3 & 3 \\
\hline $\begin{array}{l}\text { Domain } \\
\text { three: Teen } \\
\text { Club Health } \\
\text { Education }\end{array}$ & $\begin{array}{l}\text { 1. Minutes/records of the Teen Club that reflect that the } \\
\text { facilitators oriented the adolescents on the disclosure and } \\
\text { acceptance of HIV status, } \\
\text { 2. Minutes/records of the Teen Club that reflect that the } \\
\text { facilitators oriented the adolescents on adherence to } \\
\text { medication, } \\
\text { 3. Minutes/records of the Teen Club that reflect that the } \\
\text { facilitators oriented the adolescents on life skills, SRH } \\
\text { Services/stigma/discrimination? }\end{array}$ & 3 & 3 \\
\hline $\begin{array}{l}\text { Domain four: } \\
\text { Clinical Care }\end{array}$ & $\begin{array}{l}\text { 1. ALHIV have access to the clinician and a nurse for screening } \\
\text { and treatment, } \\
\text { 2. ALHIV receive ART refill during Teen Club Meeting, } \\
\text { 3. Viral Load is checked at the teen club whenever ALHIV are } \\
\text { due for viral load }\end{array}$ & 3 & 3 \\
\hline \multicolumn{2}{|c|}{$\begin{array}{lll} & \text { Total }\end{array}$} & 18 & 18 \\
\hline
\end{tabular}

A multiple logistical regression model was conducted to assess the relationships between explanatory (predictor) variables (such as age, gender, location, occupation, length of membership in Teen Club) and the outcome of interest (successful transition). Stepwise regression model employing a backward-elimination rule was used to arrive at the model that maximizes the estimation power. For qualitative data from IDI, recordings were transcribed verbatim, any identifiers were removed and pseudonyms or generic arbitrary codes were substituted. The transcripts were imported into NVIVO software for analysis.

Ethical approval for data collection for this study was provided by College of Medicine Research Ethics Committee (COMREC) in December 2018. All participants provided written, informed consent prior to data collection.

\section{Results}




\section{Study Participants' Enrollment Outcome}

Figure 3 is a summary of how data records for the retrospective cohort analyses were extracted. As shown in figure 3 , there were 2,379 ALHIV registered with Teen Clubs in the period of interest for the study in the 14 health facilities, of whom 146 were potential eligible for the study. However, we could only verify records for 131 participants across the key source documents (registers and master cards). This is because of poor record keeping characterised by use of multiple registers which had dissimilar information with incomplete records. Thus we could not achieve our desired sample size of 145 .

\section{Characteristics of Study Participants}

\section{Cohort Study}

Table 2 shows the characteristics of ALHIV enrolled in the study. The mean age of all the study participants was 19.3 (SD = 1.5). Two -thirds $(61.8 \%)$ of the total participants were female. Nearly two-thirds $(60.3 \%)$ of the participants were students and almost three quarters $(73.3 \%)$ of them were attending health facilities owned by government. The mean time spent in an HIV Teen Club for participants was 2.4 years $(S D=0.6)$. More than one third $(36.4 \%)$ of the participants were living with their parents.

\section{Table 2: Participants characteristics}

\begin{tabular}{|c|c|}
\hline Variable & $\begin{array}{l}\text { Total Enrolled } \\
(\mathrm{N}=131)\end{array}$ \\
\hline Age in years, mean (SD) & $19.3(1.5)$ \\
\hline Length of Membership in years, Mean (SD) & $2.4(0.6)$ \\
\hline \multicolumn{2}{|l|}{ Gender, $n(\%)$} \\
\hline Female & $81(61.8)$ \\
\hline Male & $50(38.2)$ \\
\hline \multicolumn{2}{|l|}{ Facility Location, $n(\%)$} \\
\hline Urban & $64(48.9)$ \\
\hline Rural & $67(51.1)$ \\
\hline \multicolumn{2}{|l|}{ Facility Ownership, n (\%) } \\
\hline Government & $96(73.3)$ \\
\hline Non-Government & $35(26.7)$ \\
\hline \multicolumn{2}{|l|}{ Occupation of adolescent $n$ (\%) } \\
\hline Student & $79(60.3)$ \\
\hline other ${ }^{1}$ & $7(5.3)$ \\
\hline Unknown ${ }^{2}$ & $45(34.4)$ \\
\hline \multicolumn{2}{|l|}{ Adolescent Guardian n (\%) } \\
\hline Biological Parents & $48(36.4)$ \\
\hline Other ${ }^{3}$ & $33(25.2)$ \\
\hline Unknown ${ }^{4}$ & $50(38.2)$ \\
\hline
\end{tabular}

Teens transitioning to adult care - "Effectiveness" dimension of the RE-AlM Framework 
Of the 131 participants who were eligible for transition between July 2017 and December 2017, only 9 (6.9\%, 95\% Cl: 2.2, 10.7) were registered to have transitioned into adult care at the same clinic, of whom seven were female. The remaining 93.6\% of the participants $(n=122)$ were still in the same facilities at the Teen Club. Only 2 the 14 health facilities (DREAM Sant'Egidio and Chikowa Health center) managed to successfully transition eligible ALHIV to adult care. The majority (8) of the transitioned ALHIV were from DREAM Sant'Egidio.

\section{Factors associated with successful transition- "Maintenance" dimension of the RE-AIM Framework}

Table 3, below, shows the results of the bivariate and multivariate logistic regression models assessing the predictors of successful transitions. In bivariate analyses, the following variables were not significantly associated with successful transition: age of the adolescent, sex of the adolescent and type of adolescent guardian. However, the following variables were significantly associated with successful transition: facility location and facility ownership. In multivariate analyses only facility location and facility ownership were significantly associated with successful transitioning. There was high collinearity between adolescent occupation and adolescent length of membership with successful transition. Therefore, these variables were omitted for further consideration in the regression modelling.

\begin{tabular}{|c|c|c|c|c|c|}
\hline Variable & $\begin{array}{l}\text { \% who } \\
\text { transitioned }\end{array}$ & $\begin{array}{l}\text { Crude OR } \\
(95 \% \mathrm{Cl})\end{array}$ & P-value & $\begin{array}{l}\text { Adjusted OR } \\
(95 \% \mathrm{Cl})\end{array}$ & p-value \\
\hline \multicolumn{6}{|l|}{ Gender } \\
\hline Male & $4.0 \%$ & 1 (Reference) & & & \\
\hline Female & $8.6 \%$ & $2.3(0.5,11.4)$ & 0.319 & - & \\
\hline \multicolumn{6}{|l|}{$\mathrm{Age}^{5}$} \\
\hline$\geq 20$ & $5.3 \%$ & 1 (Reference) & & & \\
\hline$<20$ & $7.5 \%$ & $1.5(0.3,7.4)$ & 0.460 & $\underline{ }$ & 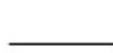 \\
\hline \multicolumn{6}{|l|}{ Facility Location } \\
\hline Urban & $12.5 \%$ & 1 (Reference) & & 1 (Reference) & \\
\hline Rural & $1.5 \%$ & $0.1(0.01,0.9)$ & 0.037 & $0.1(0.01,0.9)$ & 0.041 \\
\hline \multicolumn{6}{|l|}{ Facility Ownership } \\
\hline Government & $1.0 \%$ & 1 (Reference) & & 1 (Reference) & \\
\hline Private & $22.9 \%$ & $28.1(3.4,235.0)$ & 0.002 & $22.8(2.4,219.7$ & 0.007 \\
\hline \multicolumn{6}{|l|}{ Adolescent Guardian } \\
\hline Parents & $12.3 \%$ & 1 (Reference) & & & \\
\hline Other & $6.3 \%$ & $0.5(0.1,2.4)$ & 0.374 & 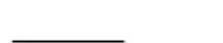 & 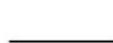 \\
\hline
\end{tabular}

\section{Implementation fidelity - "Implementation" dimension of the RE-AIM Framework}

The overall implementation fidelity of the HIV Teen Club protocols was medium (61.7\%). As shown in Figure 4 below, the clinical care domain was implemented with high fidelity $(97.6 \%)$ while the teen club operation and capacity building domains were implemented with medium fidelity ( $68.3 \%$ and $57.1 \%$, respectively). Teen Club Health Education domain registered the lowest implementation fidelity (23.8\%).

As shown in Figure 5, implementation fidelity varied widely across the 14 health facilities. Only 3 (21.4\%) facilities implemented Teen Club protocols with high fidelity. Nearly three-quarters $(71.4 \%)$ of the health facilities implemented with 
medium fidelity and 1 implemented with low fidelity. Interestingly, 2 of the 3 health facilities with high implementation fidelity successfully transition a few ALHIV to adult care, suggesting a potential association between implementation fidelity and transition outcome.

\section{Health system factors associated with successful transition - "Maintenance" dimension of the RE-AIM Framework}

\section{Qualitative study}

To further explore the challenges with operating Teen Clubs, we conducted in-depth interviews with a total of 28 SPs. These included 9 providers with nursing experience, 11 providers with a clinical experience, and 8 providers with HTS counselling background. Most participants had more than 2 years of experience working with HIV Teen Clubs. Half of the providers were trained in HIV Teen Club curriculum while only 11 (39.3\%) were trained in transitioning of adolescents from HIV Teen Club to adult care.

\section{Transitioning challenges}

The majority of providers acknowledged that they have not managed to transition adolescents to adult care as expected. The challenges were at provider, patient and health facility levels. Most providers reported that lack of provider training in the guidelines for transitioning ALHIV was main contributing factor to failure to properly transition the adolescents. Several acknowledged lack of knowledge on the guidelines for transitioning, as noted below by one provider,

"I am not sure that we have successfully transitioned these teens according to the guidelines since we have not been trained in the guidelines for transitioning the ALHIV." (Respondent \# 11).

At the patient level, the providers observed that most adolescents prefer to remain in Teen Club despite being eligible for transition according to transition guidelines, due to their perceived higher quality of care provided in Teen Clubs than in the adult ART clinics. They also reported that school-going adolescents find teen clubs to be convenient and not to interfere with schooling, as quoted below:

"A lot of kids are unwilling to be transitioned to adult care despite being eligible because of the care they receive at teen club and also they say they don't want to miss school as some are still in school and adult care appointments are always during weekdays". (Respondent \#28)

The SPs also reported human resource challenge of as a key contributing factor to successful transitioning. They reported shortage of human resource to provide specialized care which is exacerbated by the high numbers of adolescents attending Teen clubs and limited time allocated to Teen Club sessions. As lamented by one of the providers,

"The challenge we have is the inadequacy of the human resource who are trained in the teen club specifically. We have a lot of adolescents but we are just 4 nurses". (Respondent \# 9)

This challenge limits the level of interaction and communication between the provider and the teen thereby adversely affecting the adolescents' readiness to transition .

\section{Discussion}

Our study found that less than 1 on 10 (6.9\%) of eligible ALHIV enrolled in Teen Clubs successfully transitioned to adult care within six months of the expected transition time. Also, the study found medium fidelity $(61.7 \%)$ in implementation of HIV Teen Clubs' adult care transition protocols in health facilities. In addition, we noted that successful transition by ALHIV in teen clubs was significantly associated positively with facility location, while sex of the adolescent and the type of adolescent guardian were observed to be not significantly associated with successful transition. Finally, limited capacity of SPs to transition the ALHIV, refusal to transition by the ALHIV, lack of clear guidelines or tools to support transition, and overburdened human resources were identified as health system factors affecting ALHIV successful transitioning. 
Our findings of low successful transition were not consistent with previous studies[10-12, 18, 19]. which registered higher successful transition rate of at least $75 \%$. This can be attributed to differences in study population, outcomes, poor record keeping and context. Four out of five studies enrolled participants with median age of 22 years contrasting the median age of 19.3 years observed in this study. As shown in table 4, studies further varied in the definition of successful transition and the follow up period. So far 3 studies had similar definitions to that used in this study whereas 4 of the 5 studies had a follow up period of 12 months compared to 6 months follow up period used in this study. Also, all previous studies were from high income countries such as the USA and Canada with stronger health systems than Malawi. Only a few studies have been conducted in Africa, the majority of these have been qualitative focusing on exploring the perception of patients and providers with regard to barriers and facilitators of successful transition [25-33]. However, our findings of poor implementation fidelity of HIV teen club transition protocol in most health facilities suggest that our findings on low transition are valid.

Table 4: Studies on Successful transition of ALHIV to adult care

\begin{tabular}{|c|c|c|c|c|c|c|c|c|}
\hline Author & Country & $\begin{array}{l}\text { Study } \\
\text { Design }\end{array}$ & $\begin{array}{l}\text { Follow } \\
\text { up/transition } \\
\text { period }\end{array}$ & $\begin{array}{l}\text { Sample } \\
\text { size }\end{array}$ & $\begin{array}{l}\text { Median age/ } \\
\text { Age Range }\end{array}$ & Outcome Definition & Findings & Ref \\
\hline Kakkar (2016) & Canada & Cohort & 6 months & 25 & 22 yrs & $\begin{array}{l}\text { Retention ( } \geq 1 \text { clinic visit } \\
\text { within } 6 \text { months of } \\
\text { interview) }\end{array}$ & $\begin{array}{l}76 \% \text { transitioned (a mean of } \\
3.6 y r s \text { after transition from } \\
\text { paediatric to adult care) }\end{array}$ & {$[12]$} \\
\hline Hussen (2017) & USA & Cohort & 12 months & 72 & 25 yrs & $\begin{array}{l}\text { Retention ( } \geq 2 \text { visits } \geq 3 \\
\text { months apart) }\end{array}$ & $\begin{array}{l}89 \% \text { retained in first year after } \\
\text { transition to adult care. }\end{array}$ & {$[18]$} \\
\hline Ryscavage (2016) & USA & Cohort & 12 months & 50 & $\begin{array}{l}\text { Perinatal 22yrs } \\
\text { Horizontal 25yrs }\end{array}$ & $\begin{array}{l}\text { Retention ( } \geq 2 \text { visits over } \\
12 \text { months following } \\
\text { linkage into adult care }\end{array}$ & $\begin{array}{l}58 \% \text { transitioned to into adult } \\
\text { care at } 12 \text { months. }\end{array}$ & {$[10]$} \\
\hline Izzo (2017) & Italy & Cohort & 12 months & 24 & $\begin{array}{l}18 \text { when } \\
\text { transitioning }\end{array}$ & Retention (not defined) & $\begin{array}{l}100 \% \text { retained at } 12 \text { months } \\
\text { post-transition }\end{array}$ & [19] \\
\hline Maturo (2015) & USA & Cohort & 12 months & 38 & 24.66 & $\begin{array}{l}\text { Successful transition: } \\
\text { One-year follow-up is } \\
\text { conducted with client by } \\
\text { psychosocial team. }\end{array}$ & $\begin{array}{l}47 \% \text { transitioned in care after } \\
\text { transition }\end{array}$ & {$[11]$} \\
\hline
\end{tabular}

Our multivariate analyses found that ALHIV attending HIV teen club in health facilities located in rural areas were less likely to transition into adult care compared to ALHIV attending HIV teen club in health facilities located in urban areas while those attending HIV teen club in non-government health facilities were more likely to transition to adult care than those attending in government health facilities. We found only 2 health centres successfully transitioned to adult care and almost all ALHIV who successfully transitioned to adult care came from one non-government facility located in urban area.

We observed that lack of training in transitioning ALHIV, lack of clear guidelines or tools to support transition, inadequate human resource and refusal of ALHIV to transition to adult care negatively influenced successful transition. These findings were consistent with other findings that explored provider's perspective on ALHIV transition[25-33]. Studies from Uganda and South Africa[31, 32] found that training, lack of clear guidelines or tools to support transition and overburdened SPs affected successful transition[28-30]. Similar findings were also noted in studies from Dominican Republic and USA which further highlighted refusal by ALHIV to transition to adult care as another challenge facing the transition process[33, 34]. 
The major strength of this study is that it was able to triangulate findings using different methodologies. This approach facilitated the validation of quantitative findings (low transitioning) by qualitative findings and also added depth on other reasons for poor transitioning (adolescent reluctance). One limitation of the study was few factors were available to analyse their relationship with successful transition, since the study design was retrospective. In addition, only a few ALHIV transitioned and this may have limited the power of our study to identify factors that were associated with successful transition. In our study, poor record keeping in health facilities may have prevented us from identifying adolescents who have successfully transitioned within the same health facility or elsewhere. Retrospective cohort study suffers from this challenge. The study did not interview ALHIV or their guardians to get their perspectives on their experiences attending Teen Clubs and barriers and facilitators of transitioning to adult care. The study focussed on interviewing health services providers and analysing clinic records. Future studies need to get perspectives of service users.

\section{Implications of the findings to clinical care or public health}

The study findings highlight the need to improve HIV health care transition for ALHIV in Malawi. The observed challenge of ALHIV transition to adult care, in particular the refusal of ALHIV to transition, reveals the current challenge in health care provision and the need to educate ALHIV, train HCW in transition ALHIV in Teen Clubs, train ART providers to manage newly transitioned adolescents and improve the quality of ART clinics to make them suitable for young people. The findings also point to the need for clear guidelines for transitioning ALHIV to adult care. This will need to be addressed in tandem with capacity-building in the guidelines. Furthermore, the results indicate that the quality of clinical data needs to be improved to better monitor patients accurately during their transition within or outside the health facility. The results also reveal the need to improve service quality, particularly fidelity in teen education implementation

\section{Conclusions}

Transitioning of ALHIV remains a challenge with many preferring to remain in pediatric care which seem to offer the best care for them. Poor implementation of transition protocols and low human resource capacity need to be addressed to improve successful transition of ALHIV to adult care.

\section{Abbreviations}

AIDS Acquired Immune Deficiency Syndrome

ALHIV Adolescents Living with HIV

ART Antiretroviral Therapy

COMREC College of Medicine Research and Ethics Committee

EMR Electronic Medical Records

DSD Differentiated Service Delivery

HIV Human immunodeficiency virus

IDI In-Depth Interviews

RE-AIM Reach, Effectiveness, Adoption, Implementation, Maintenance,

SD Standard Deviation

\section{Declarations}


Ethics approval and consent to participate

The study protocol was reviewed and approved by the College of Medicine Research and Ethics Committee (COMREC) under research certificate number P.09/18/2481. Permissions to carry out the study was obtained from the District Health Officer (DHO) for Blantyre.

\section{Consent for publication}

All participants consented for peer review and the publication of the study results.

\section{Availability of data}

Data for this study can be accessed at University of Malawi College of Medicine Library and from the corresponding author upon request.

\section{Competing interests}

All authors have no competing interest to declare.

\section{Funding}

This study was supported by Malawi HIV Implementation Science Research Training

\section{Authors' contributions}

JG designed the study, oversaw data collection, analysed the data and wrote the paper. VM reviewed the manuscript and approved the final version for submission.

\section{Acknowledgements}

We would like to thank all the study participants who took time to take part in the interviews and the health facilities where data was collected.

\section{References}

1. UNICEF. Tuming the tide against AIDS will require more concentrated focus on adolescents and young people 2018 [22 June 2018]. Available from: https://data.unicef.org/topic/hivaids/adolescents-young-people/\#.

2. UNAIDS. The Gap Report2014 [2 May 2018]:[422 p.]. Available from: http://files.unaids.org/en/media/unaids/contentassets/documents/unaidspublication/2014/UNAIDS_Gap_report_en.pdf.

3. Malawi National Statistical Office. Malawi Demographic and Health Survey 2015-16. Zomba Malawi, and Rockville, Maryland, USA2017.

4. UNAIDS. Country Factsheet Malawi 2016 [12 June 2018]. Available from: http://www.unaids.org/en/regionscountries/countries/malawi/.

5. Ministry of Health Malawi. First Malawi Population-based HIV Impact Assessment (MPHIA) 2015-16:Report. November 2017. Report No.: 1.

6. Ministry of Health Malawi. National Youth Friendly Health Services Strategy 2015-2020 2015.

7. WHO. HIV and adolescents: guidance for HIV testing and counseling and care for adolescents living with HIV: recommendations for a public health approach and considerations for policy-makers and managers2013 [26 June 2018].

8. Hussen SA, Chahroudi A, Boylan A, Camacho-Gonzalez AF, Hackett S, et al. Transition of youth living with HIV from pediatric to adult-oriented healthcare: a review of the literature. Future virology [Internet]. 2015 [1 July 2018]; 9(10):[921-9 pp.]. Available from: http://www.ncbi.nlm.nih.gov/pubmed/25983853. 
9. Castelnuovo B, Mubiru F, Nakalema S, Twimukye A, Kiragga A. Describing the retention in care of human immunodeficiency virus-positive young adults who transition from adolescent to adult care. International health. 2018;10(4):318-20.

10. Ryscavage P, Macharia T, Patel D, Palmeiro R, Tepper V. Linkage to and retention in care following healthcare transition from pediatric to adult HIV care. AIDS care [Internet]. 2016 [15 July 2018]; 28(5):[561-5 pp.]. Available from: http://www.ncbi.nlm.nih.gov/pubmed/26766017.

11. Maturo D, Powell A, Major-Wilson H, Sanchez K, De Santis JP, et al. Transitioning Adolescents and Young Adults With HIV Infection to Adult Care: Pilot Testing the "Movin' Out" Transitioning Protocol. Journal of pediatric nursing. 2015;30(5):e2935.

12. Kakkar F, Van der Linden D, Valois S, Maurice F, Onnorouille M, et al. Health outcomes and the transition experience of HIV-infected adolescents after transfer to adult care in Quebec, Canada. BMC pediatrics. 2016;16:109.

13. Nachega JB, Marconi VC, van Zyl GU, Gardner EM, Preiser W, et al. HIV treatment adherence, drug resistance, virologic failure: evolving concepts. Infectious disorders drug targets. 2011;11(2):167-74.

14. HIV LEARNING NETWORK. The CQUIN Learning Network Differentiated Service Delivery for Adolescents Living with HIV: Meeting Summary. Johannesburg, South Africa: ICAP, 2017.

15. WHO. Differentiated service delivery for families - children, adolescents, and pregnant and breastfeeding women: A background review2016 [3 July 2018]. Available from:

https://www.differentiatedcare.org/Portals/0/adam/Content/QLbAMeHLEkCehaXD45xXQ/File/DSD\%20Families\%20review_28Nov.pdf.

16. Baylor College of Medicine Children's Foundation. Adolescent HIV And Psychosocial Support: A Teen Club Training Manual and Resource Handbook. 2015.

17. MacKenzie RK, van Lettow M, Gondwe C, Nyirongo J, Singano V, et al. Greater retention in care among adolescents on antiretroviral treatment accessing "Teen Club" an adolescent-centred differentiated care model compared with standard of care: a nested case-control study at a tertiary referral hospital in Malawi. Journal of the International AIDS Society. 2017;20(3):e25028.

18. Hussen SA, Chakraborty R, Knezevic A, Camacho-Gonzalez A, Huang E, et al. Transitioning young adults from paediatric to adult care and the HIV care continuum in Atlanta, Georgia, USA: a retrospective cohort study. Journal of the International AIDS Society [Internet]. 2017 [1 September 2018]; 20(1):[21848 p.]. Available from:

http://www.ncbi.nlm.nih.gov/pubmed/28872281 https://onlinelibrary.wiley.com/doi/pdf/10.7448/IAS.20.1.21848.

19. Izzo I, Quiros-Roldan E, Saccani B, Chiari E, Casari S, et al. Perinatally HIV-Infected Youths After Transition from Pediatric to Adult Care, a Single-Center Experience from Northern Italy. AIDS research and human retroviruses. 2018;34(3):241-3.

20. Zanoni BC, Sibaya T, Cairns C, Lammert S, Haberer JE. Higher retention and viral suppression with adolescent-focused HIV clinic in South Africa. PloS one. 2017;12(12):e0190260.

21. Cochran WG. Sampling Techniques. Unitend States of America, New York.: Wiley and Sons, Inc; 1963.

22. Glasgow RE, Vogt TM, Boles SM. Evaluating the public health impact of health promotion interventions: the RE-AIM framework. American Journal of Public Health. 1999;89(9):1322-7.

23. Glasgow R, E., , Vogt T, M, Boles SM. Evaluating the Public Health Impact of Health Promotion Interventions: The RE-AIM Framework. 1999.

24. Ryscavage P, Tepper V. Clinical Outcomes Following Transition from Pediatric to Adult HIV Care 2013 [22 July 2018]. Available from: https://idsa.confex.com/idsa/2013/webprogram/Paper39557.html.

25. Mark D, Armstrong A, Andrade C, Penazzato M, Hatane L, et al. HIV treatment and care services for adolescents: a situational analysis of 218 facilities in 23 sub-Saharan African countries. Journal of the International AIDS Society. 2017;20(Suppl 3):21591.

26. Dahourou DL, Gautier-Lafaye C, Teasdale CA, Renner L, Yotebieng M, et al. Transition from paediatric to adult care of adolescents living with HIV in sub-Saharan Africa: challenges, youth-friendly models, and outcomes. Journal of the 
International AIDS Society. 2017;20(Suppl 3):21528.

27. Kung TH, Wallace ML, Snyder KL, Robson VK, Mabud TS, et al. South African healthcare provider perspectives on transitioning adolescents into adult HIV care. South African Medical Journal. 2016;106(8):804.

28. Gillespie SP, ED; Anabwani, GM; Hlatshwayo, M; Kazembe, PN; Mohapi, EQ; , et al. Healthcare transition practices across the Baylor College of Medicine International Pediatric AIDS Initiative (BIPAI) Network of pediatric HIV clinics. 8th International Workshop on HIV Pediatrics; Durban, South Africa2016.

29. Rakhmanina NC, B; Kose, J; Manson, K. . New Horizons Advancing Pediatric HIV Care Collaborative. Approaches to care for the HIV-infected adolescents across national HIV/AIDS programs participating in the New Horizons advancing paediatric HIV care collaborative. 21st International AIDS Conference; Durban, South Africa2016.

30. Frigati L, Brittain K, Rabie H, Cotton M, Joubert S, et al. Diversity in adolescent HIV care services in the public sector in Cape Town, South Africa. 8th International Workshop on HIV Pediatrics; Durban, South Africa2016.

31. Chekata Inzaule S, Hamers R, Kityo C, Rinke de Wit T, Roura M. Barriers to antiretroviral treatment adherence in HIVinfected long-term treated adolescents and adults in Uganda. 21st International AIDS Conference; Durban, South Africa2016.

32. Willis NM, M; Dupwa, B; Ncube, G; Murungu, J; Mugurungi, O. . Transitioning adolescents with HIV - the Zimbabwean perspective. 8th International Workshop on HIV Pediatrics; Durban, South Africa2016.

33. Pinzon-Iregui MC, Ibanez G, Beck-Sague C, Halpern M, Mendoza RM. "...like because you are a grownup, you do not need help": Experiences of Transition from Pediatric to Adult Care among Youth with Perinatal HIV Infection, Their Caregivers, and Health Care Providers in the Dominican Republic. Journal of the International Association of Providers of AIDS Care. 2017;16(6):579-87.

34. Tepper V, Zaner S, Ryscavage P. HIV healthcare transition outcomes among youth in North America and Europe: a review. Journal of the International AIDS Society. 2017;20(Suppl 3):21490.

\section{Figures}




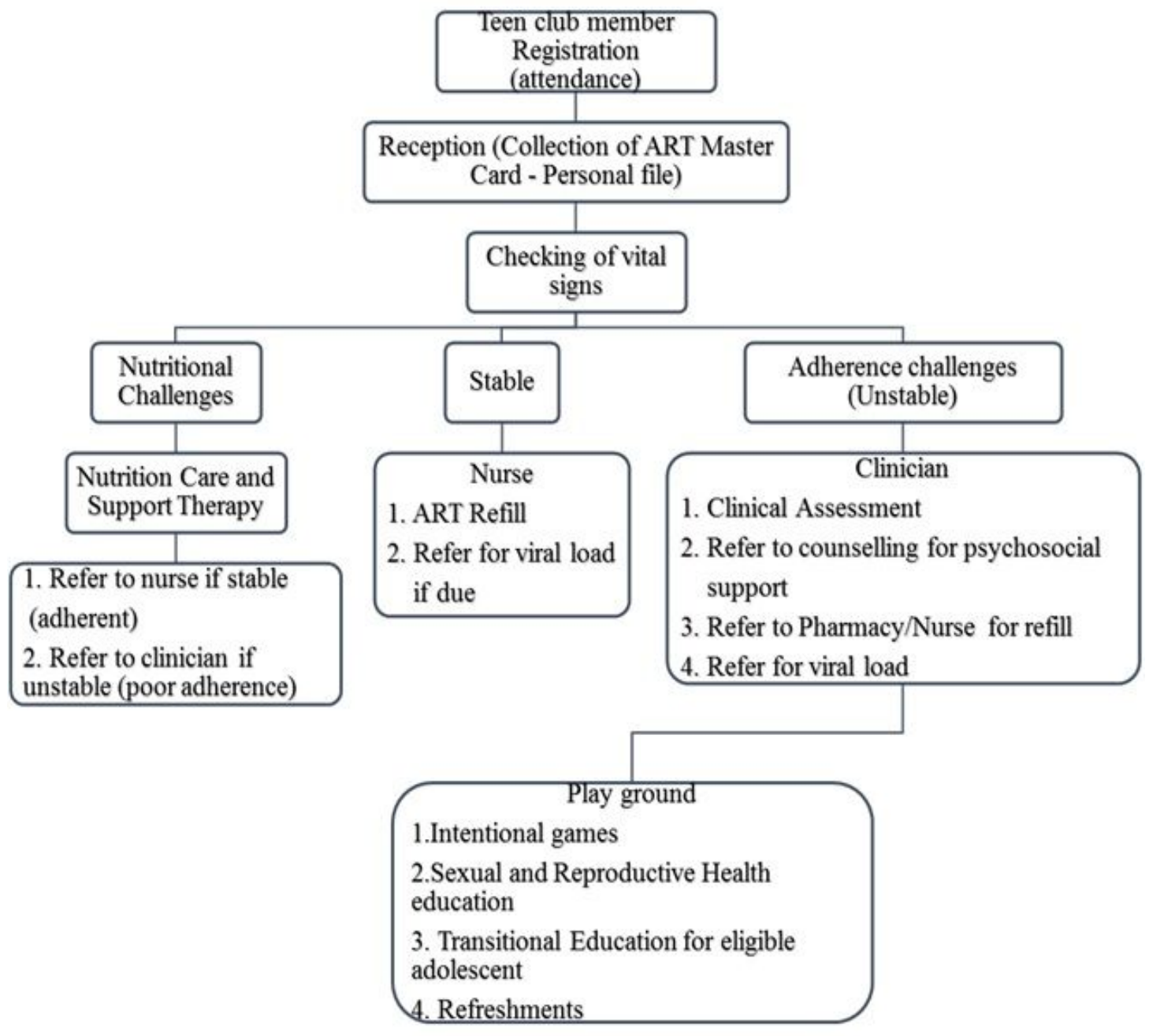

Figure 1

Teen Club model: Session flow chart

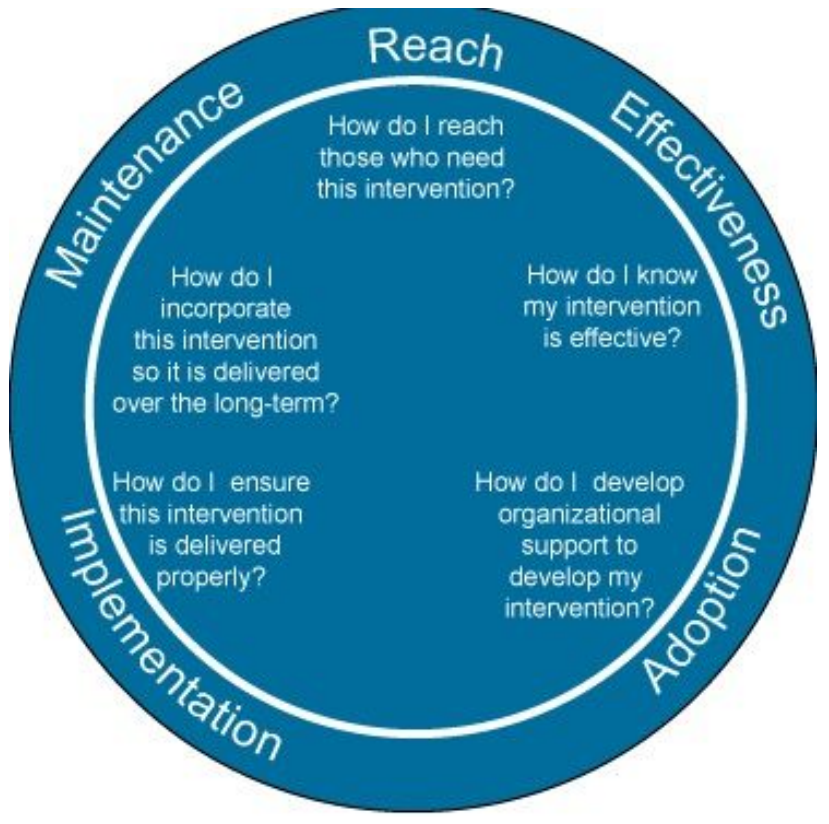




\section{Figure 2}

RE-AIM Framework Illustration

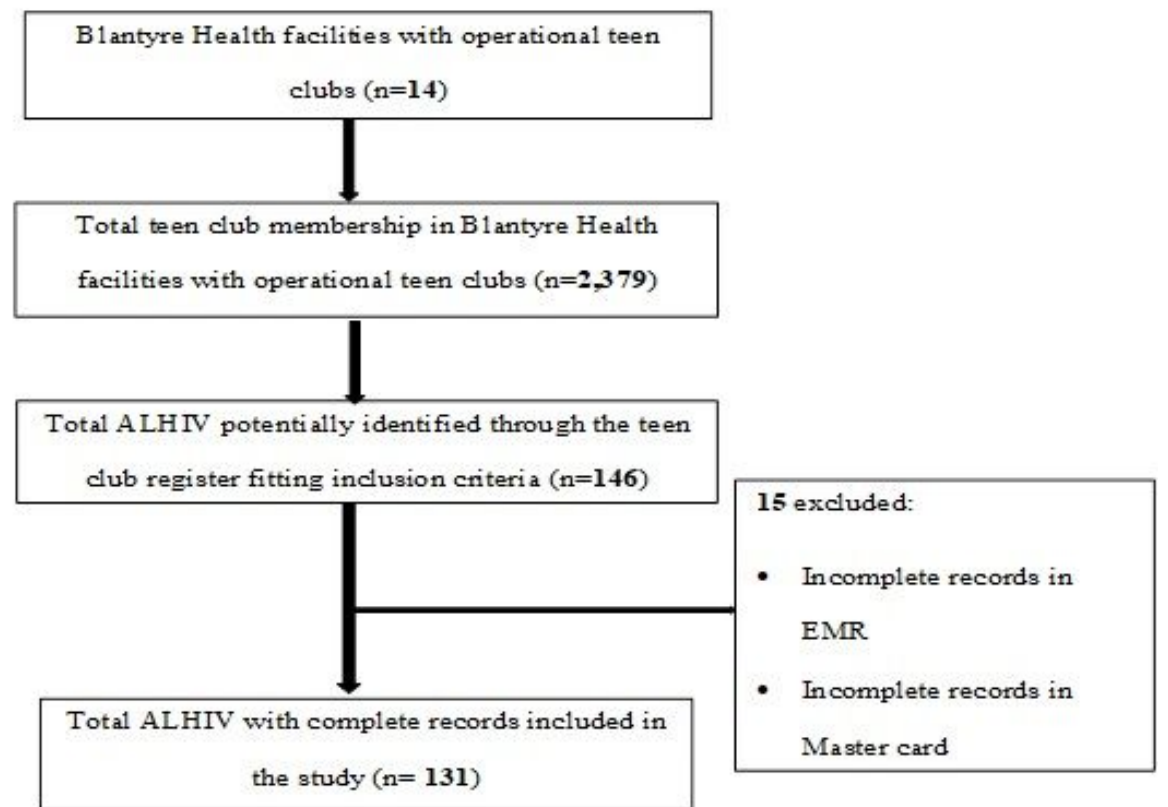

\section{Figure 3}

Data extraction flow chart

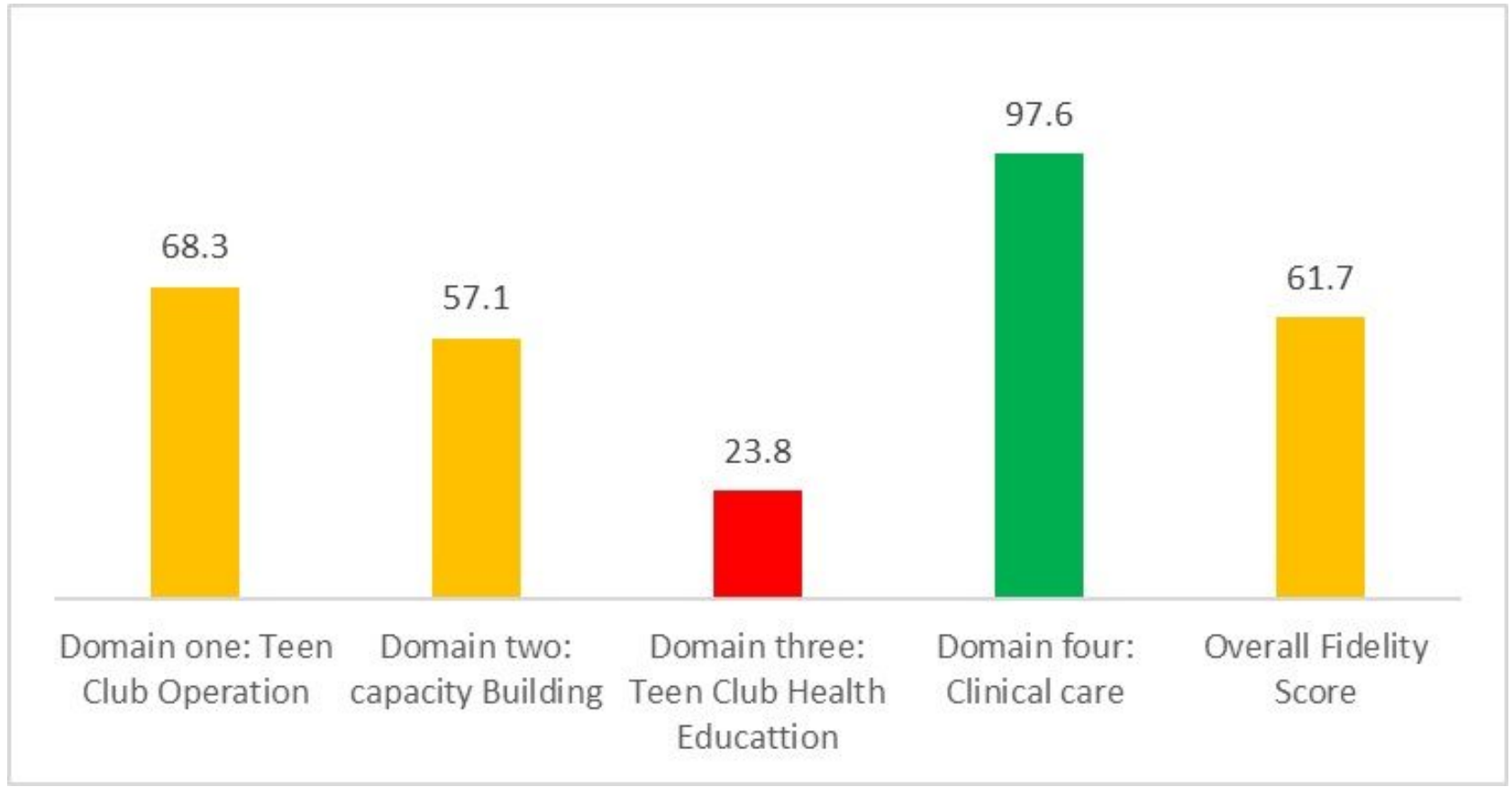

\section{Figure 4}

Fidelity Score per Domain 


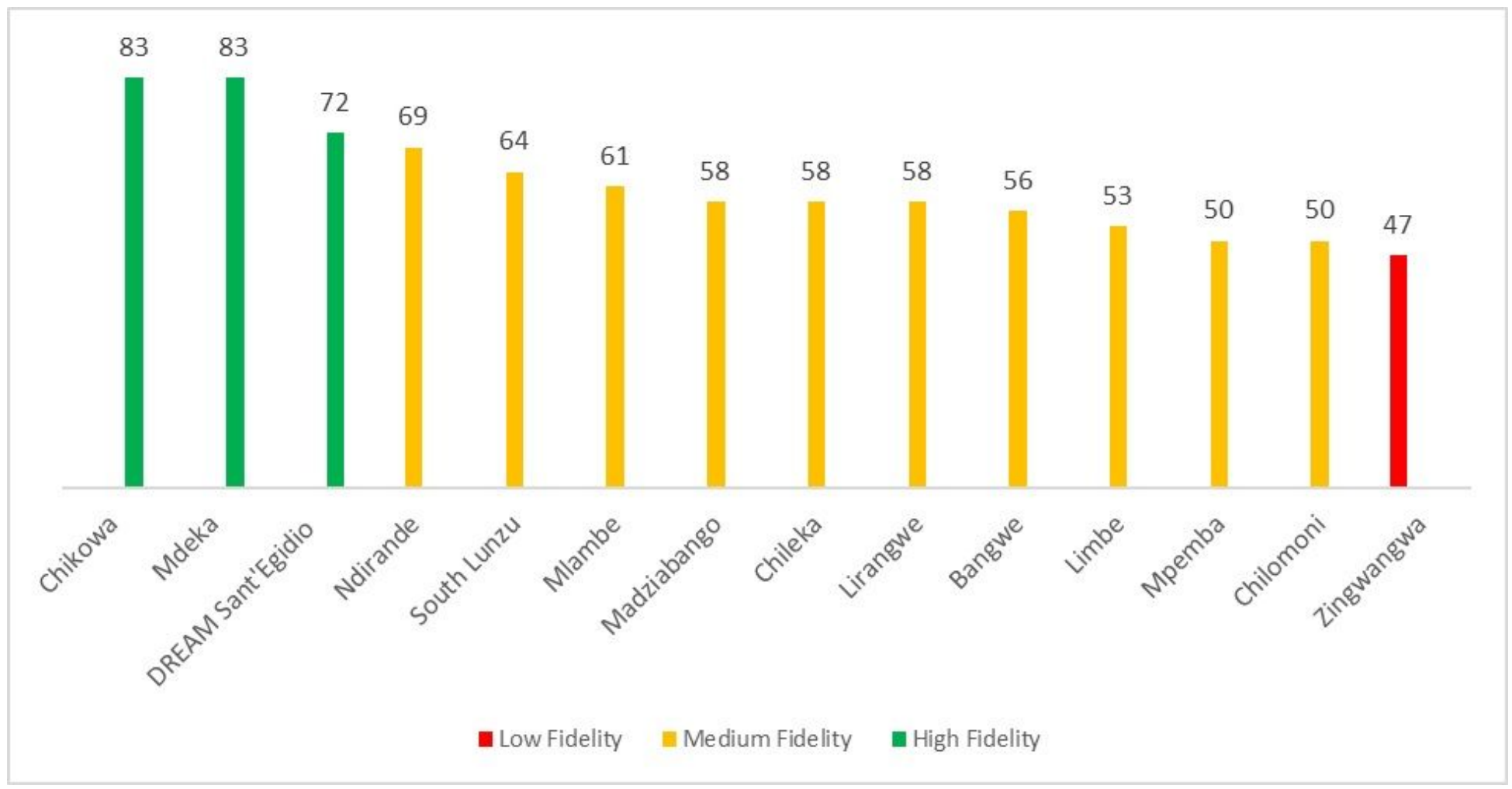

Figure 5

Fidelity Score per health facility 